\title{
INFLUÊNCIA DO TEMPO DE REVESTIMENTO NO TAMANHO E ESTABILIDADE DE NANOPARTICULAS DE MAGNETITA PARA TRATAMENTOS DE HIPERTERMIA MAGNÉTICA
}

\author{
Mara Carolina do Carmo Paresque ' \\ Elizabeth Mendes de Oliveira ${ }^{2}$ \\ José Adilson de Castro '
}

\section{Resumo}

Em tratamentos de hipertermia magnética, as etapas de produção e revestimento das nanopartículas magnéticas são de fundamental importância para sua funcionalidade e eficiência durante a dinâmica do tratamento. $O$ método de coprecipitação vem sendo utilizado para sintetizar nanopartículas de magnetita $\left(\mathrm{Fe}_{3} \mathrm{O}_{4}\right)$, largamente utilizadas neste tratamento. As mesmas foram recobertas com uma camada polimérica de polietilenoglicol (PEG) visando prevenir a oxidação do núcleo, permitir sua dispersão em água, aumentar sua estabilidade coloidal, bem como evitar a aglomeração das nanopartículas. Foram realizadas cinco sínteses, cada uma delas com tempos da etapa de revestimento das nanopartículas de 10, 20, 30, 50 e 60 minutos, respectivamente. Foram avaliadas a influência do tempo de revestimento no tamanho das partículas utilizando Nanoparticle Traking Analysis (NTA) e a estabilidade do revestimento por TGA/DSC. As nanopartículas revestidas apresentaram diâmetros médios na faixa de $3 \mathrm{I}$ a $40 \mathrm{~nm}$. Analises no TGA/DSC permitiram observar a presença de uma camada adsorvida na superfície do núcleo magnético, bem como a eficácia do polietilenoglicol na proteção do núcleo de magnetita.

Palavras-chave: Nanopartículas magnéticas; Magnetita; Polietilenoglicol; Tempos de revestimento.

\section{INFLUENCE OF THE COATING TIME ON THE SIZE AND STABILIZATION OF MAGNETITE NANOPARTICLES FOR MAGNETIC HYPERTHERMIA TREATMENT}

\begin{abstract}
In the magnetic hyperthermia treatment the nanoparticles production and coating procedure are fundamental for the efficiency during the treatment dynamics. The coprecipitation method has been used to synthesize magnetite nanoparticles (Fe3O4). They were coated with a polyethylene glycol (PEG) polymer layer to prevent the oxidation of the magnetic core, to allow dispersion of the nanoparticles in water, to increase their colloidal stability, as well as to avoid the agglomeration of the particles. Five syntheses were carried out with the coating stage of the particles times of I0,20,30,50 and 60 minutes, aiming to evaluate the influence of coating time on the final nanoparticle size using Nanoparticle Traking Analysis (NTA) measurements and coating stability using TGA/DSC techniques. The results indicated that average diameters in the range of $3 \mathrm{I}$ to $40 \mathrm{~nm}$ were obtained. TGA/DSC results indicated that a stable adsorbed layer on the surface of the magnetic core promoted by the polyethylene glycol is effective in the protection of the magnetite nucleus.
\end{abstract}

Keywords: Magnetic nanoparticles; Magnetite; Polyethylene glycol; Coating times.

\section{INTRODUÇÃO}

$\mathrm{Na}$ atualidade existe um crescente interesse no estudo e desenvolvimento de materiais em escala nanométrica, em particular aos materiais que apresentam propriedades melhoradas provenientes de suas características únicas apresentadas em escala manométrica. Devido a características únicas tais como reduzido tamanho e superfícies ativas tem-se materiais magnéticos, óticos, eletrônicos, dentre outros que apresentam aplicações de alta eficiência [I].

'Universidade Federal Fluminense - UFF, Volta Redonda, RJ, Brasil. E-mail: paresquemara@gmail.com

${ }^{2}$ Centro Federal de Educação Tecnológica Celso Suckow da Fonseca - CEFET, Angra dos Reis, RJ, Brasil

2176-I523 (C) 2019 Associação Brasileira de Metalurgia, Materiais e Mineração. Published by ABM. This is an open access paper, published under the Creative Commons CC BY-NC-ND license (Attribution-NonCommercial-NoDerivs) - https://creativecommons.org/licenses/ by-nc-nd $/ 4.0 \%$. 
Tais nanopartículas podem ser potencialmente utilizadas nos mais diversos campos como eletrônica, ótica, aeroespacial, medicamentos, células combustíveis, além também de geração e armazenamento de energia que lhe são conferidas tais especialidades devido a suas propriedades intrínsecas e desenvolvidas através de funcionalidades superficiais.

Nesta última década, as nanopartículas (NPs) de óxido de ferro representam um viés interessante nas diversas aplicações biomédicas, sendo amplamente estudadas. Nesse sentido, tais nanopartículas se sobressaem devido ao seu comportamento quimicamente estável, não tóxico e biocompatível [2,3] As nanopartículas de magnetita $\left(\mathrm{Fe}_{3} \mathrm{O}_{4}\right)$, em especial, fazem parte de uma classe denominada nanopartículas magnéticas (MNPs), e se destacam por apresentar um comportamento único como o superparamagnetismo, alta coercitividade, baixa temperatura de Curie, e também uma elevada susceptibilidade magnética [4].

As MNPs de magnetita podem ser obtidas de diversas formas tais como coprecipitação química, síntese hidrotérmica, decomposição térmica, micro-ondas, síntese eletroquímica, ultrassom dentre outras, entretanto, a técnica de coprecipitação química de sais de ferro ainda é a mais utilizada em virtude da sua simplicidade e do seu baixo custo. No entanto, devido à grande área superficial e as fortes interações dipolo-dipolo apresentadas pelas nanopartículas magnéticas tornam-se imprescindíveis que suas superfícies sejam funcionalizadas visando melhorar a estabilização, evitar sua aglomeração, bem como aumentar o tempo de circulação no sistema $[5,6]$.

Entretanto, o núcleo de magnetita pode ser revestido com uma camada de surfactantes, polímeros ou materiais inorgânicos tornando assim as nanopartículas hidrossolúveis e biocompatíveis. Exemplos de substâncias utilizadas para revestir a superfície das partículas são Gelatina, Dextrano, Polivinil álcool (PVA), Polietilenoglicol (PEG), Quitosano, etc $[7,8]$. Em aplicações biomédicas, tão importante quanto o revestimento da superfície magnética das nanopartículas são suas características morfológicas, seu tamanho, sua distribuição granulométrica e sua magnetização $[9,10]$, características estas que se relacionam diretamente ao processo de síntese das partículas, bem como o controle de suas condições de processamento (temperatura, atmosfera, $\mathrm{pH}$, sais utilizados, etc) interferem diretamente no produto final obtido $[11,12]$. Uma outra vertente das diversas aplicações, tem-se a integração das áreas de terapia e diagnóstico como agentes de contraste de ressonância magnética (MRI), bem como veículos na entrega controlada de fármacos na terapia de tumores, bioseparação e biosensores, hipertermia magnética, engenharia de tecidos $[13,14]$.

A terapia de hipertermia pode ser empregada no tratamento de câncer, tornando-se especialmente útil em casos nos quais os tumores oferecem algum tipo de resistência à quimioterapia, sendo utilizada então para aumentar a eficácia do tratamento ao ser combinada com quimioterapia ou radioterapia [15]. Para tanto, as MNPs são direcionadas a uma região alvo doente e em seguida são submetidas a um campo magnético alternado que torna possível para as partículas converterem a energia eletromagnética em calor, gerando assim o aquecimento de uma região específica na faixa de $42-46^{\circ} \mathrm{C}$ necessário para danificar as células cancerígenas $[16,17]$ minimizando os danos sobre o tecido saudável.

Nesse trabalho, nanopartículas de magnetita $\left(\mathrm{Fe}_{3} \mathrm{O}_{4}\right)$ foram sintetizadas utilizando a técnica de coprecipitação de sais de ferro visando um melhor controle das condições de síntese. As partículas foram revestidas com Polietilenoglicol (PEG) com diferentes tempos de duração do processo de revestimento e em seguida foram caracterizadas através das técnicas de TGA/DSC para avaliar a qualidade e a capacidade de proteção da camada polimérica utilizada para recobrir o núcleo magnético e Nanoparticle Traking Analysis (NTA) visando avaliar a distribuição granulométrica das partículas obtidas através da síntese da magnetita.

\section{MATERIAIS E MÉTODOS}

\section{I Materiais}

Tanto o cloreto férrico P.A. $\left(\mathrm{FeCl}_{3} \cdot 6 \mathrm{H}_{2} \mathrm{O}\right)$ como o sulfato ferroso heptahidratado P.A. $\left(\mathrm{FeSO}_{4} \cdot 7 \mathrm{H}_{2} \mathrm{O}\right)$ foram obtidos da Reagen e o hidróxido de amônio P.A. $\left(\mathrm{NH}_{4} \mathrm{OH}\right)$ e o Polietilenoglicol 4000 (PEG) foram obtidos da Synth. Água ultrapura milli-Q foi utilizada em todas as etapas do processo.

Foi feita uma base contendo $50 \%$ de hidróxido de amônio P.A. $\left(\mathrm{NH}_{4} \mathrm{OH}\right)$ e $50 \%$ de água milli-Q a fim de ser posteriormente utilizada na precipitação da magnetita.

\subsection{Métodos}

\subsection{Síntese da magnetita}

O processo de síntese das nanopartículas de magnetita, exemplificado na Figura I, teve início com o aquecimento de $200 \mathrm{ml}$ de água ultrapura visando reduzir a quantidade de oxigênio presente no meio reacional e prevenir a oxidação dos íons de ferro utilizados.

O controle do tamanho, forma e composição das nanopartículas depende diretamente do tipo de sais utilizados, da razão de $\mathrm{Fe}^{2+}$ e $\mathrm{Fe}^{3+}$, do $\mathrm{pH}$, da temperatura do meio reacional entre outras coisas [13]. Dessa forma, foi feita a dissolução, sob agitação magnética, do sulfato ferroso $(\mathrm{I}, 18 \mathrm{~g})$ e do cloreto férrico $(2,025 \mathrm{~g})$ em meio aquoso a uma temperatura de $70^{\circ} \mathrm{C}$ obedecendo a proporção molar I: $2\left(\mathrm{Fe}^{2+}: \mathrm{Fe}^{3+}\right)$. Decorridos 10 minutos, adicionou-se diretamente ao meio reacional a base contento $o$ agente precipitante $\mathrm{NH}_{4} \mathrm{OH}$ através de gotejamento podendo-se então observar a formação de uma solução de coloração preta (precipitação da magnetita).

Após ocorrer a precipitação, foi iniciado o processo de funcionalização da superfície das nanopartículas utilizando polietilenoglicol (PEG) com o intuito de criar uma barreira 


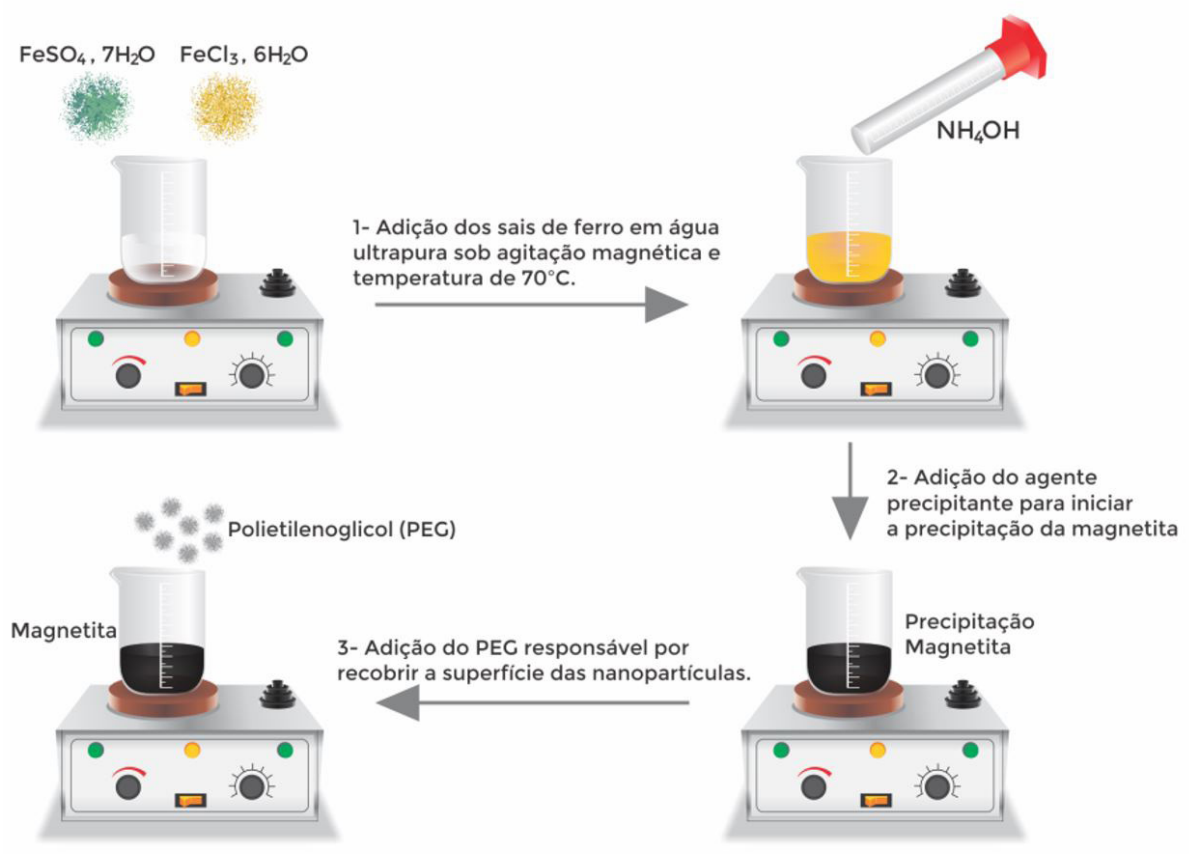

Figura I. Esquema da síntese da magnetita.

entre o núcleo das NPs e o ambiente, tormar as partículas hidrossolúveis, além de proteger e estabilizar o núcleo de ferro. O revestimento das MNPs foi feito durante a síntese. Para isso, após a precipitação da magnetita, foram acrescentados $4 \mathrm{~g}$ de polietilenoglicol ao meio reacional. Essa mistura foi mantida sob agitação magnética constante e moderada durante tempos distintos para cada amostra.

As cinco amostras foram revestidas por 10,20,30, 50 e por fim 60 minutos. Em seguida, cada amostra foi lavada com água ultrapura até $\mathrm{opH}$ da solução atingir um valor igual a 10. As nanopartículas presentes na solução foram então recuperadas por decantação magnética, acomodadas em placas de Petri e levadas para secagem em estufa a $40^{\circ} \mathrm{C}$ por um período de 24 horas. Após a secagem, as nanopartículas foram maceradas em um almofariz de porcelana e encaminhadas à caracterização.

\subsubsection{Caracterização}

As amostras foram submetidas a análises termogravimétricas no equipamento TGA/DSC modelo SDT Q600 da TA Instruments visando avaliar as interações entre o núcleo magnético e a camada de revestimento, bem como sua estabilidade mediante temperaturas elevadas.

Os ensaios foram realizados a uma taxa de aquecimento constante de $10^{\circ} \mathrm{C} / \mathrm{min}$ e uma vazão de $100 \mathrm{ml} / \mathrm{min}$ de gás nitrogênio criando uma atmosfera inerte prevenindo a oxidação das nanopartículas em contato com o ar. Durante cada ensaio foram mantidas isotermas de 10 minutos de duração em cada um dos patamares de temperatura nos quais ocorrem os inícios das transformações de fase, sendo eles $100^{\circ} \mathrm{C}, 300^{\circ} \mathrm{C}$ e $700^{\circ} \mathrm{C}$.

O equipamento NTA 2.3 foi utilizado para obter as distribuições granulométricas das amostras, o tamanho médio dos cristais e os diâmetros de corte para 10, 50 e 90\%. Para tanto, as amostras foram dispersas em água ultrapura, sem adição de tensoativo e respeitando um fator de diluição de $1: 100(\% \mathrm{p} / \mathrm{v})$. As soluções foram acondicionadas em frascos de Erlenmeyer e mantidas sob agitação vigorosa por 24 horas com 250 rpm (Mesa Agitadora Tecnal). Após esse período foi feita a analise da cada solução utilizando o NTA.

\section{RESULTADOS}

Os ensaios realizados utilizando o analisador de partículas NTA mostraram diâmetros médios variando de 3 I a $40 \mathrm{~nm}$, conforme a Tabela I.

O tamanho médio das partículas se encontra dentro da faixa esperada para aplicações biomédicas que está entre 10-50 nm, possibilitando dessa forma uma capacidade de resposta satisfatória quando expostas a um campo magnético externo, geração de calor para técnica de hipertermia, além de um bom equilíbrio dos tempos de circulação no organismo, devido também à presença do revestimento adequado [18]. Ainda segundo Medeiros, partículas menores do que $10 \mathrm{~nm}$ apresentam uma resposta inferior aos campos magnéticos externamente aplicados em função da sua magnetização de saturação reduzida, sendo a faixa de tamanho ideal para aplicação em hipertermia entre 10 e $20 \mathrm{~nm}$. 
Tabela I. Distribuição granulométrica das nanopartículas de óxido de ferro

\begin{tabular}{ccccc}
\hline $\begin{array}{c}\text { Tempos de } \\
\text { Revestimento }\end{array}$ & Tamanho Médio (nm) & DI0 (nm) & D50 (nm) & D90 (nm) \\
\hline 10 minutos & 31 & 14 & 24 & 53 \\
20 minutos & 33 & 19 & 28 & 50 \\
30 minutos & 40 & 22 & 34 & 63 \\
50 minutos & 40 & 22 & 32 & 63 \\
60 minutos & 38 & 21 & 59 \\
\hline
\end{tabular}

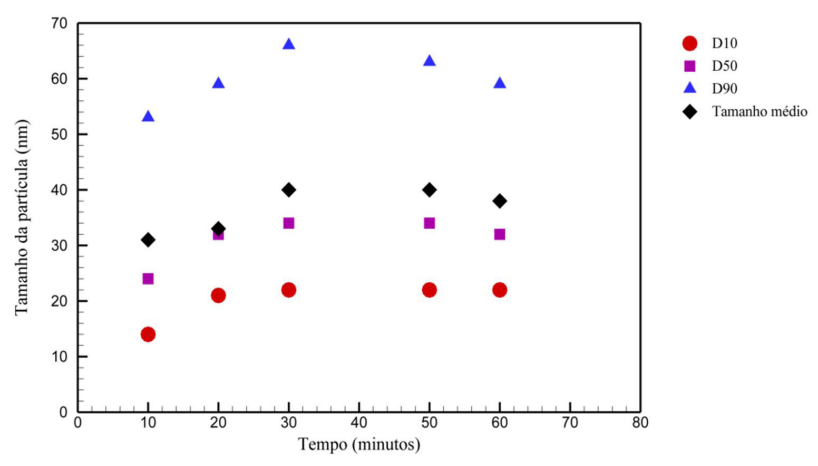

Figura 2. Distribuição granulométrica das nanopartículas de óxido de ferro.

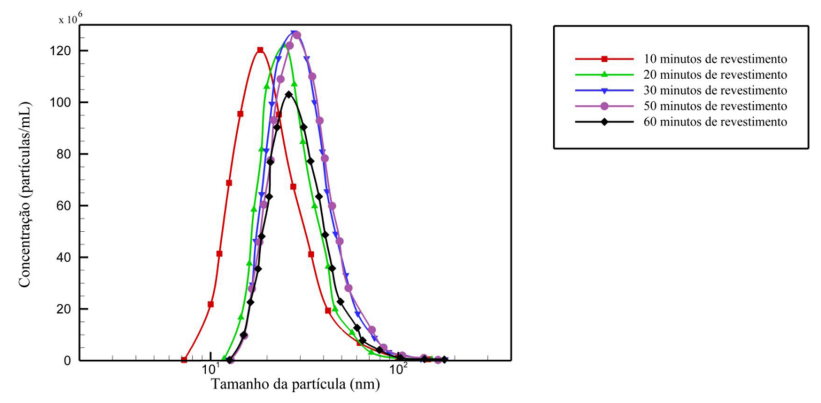

Figura 3. Concentração das nanopartículas de magnetita.

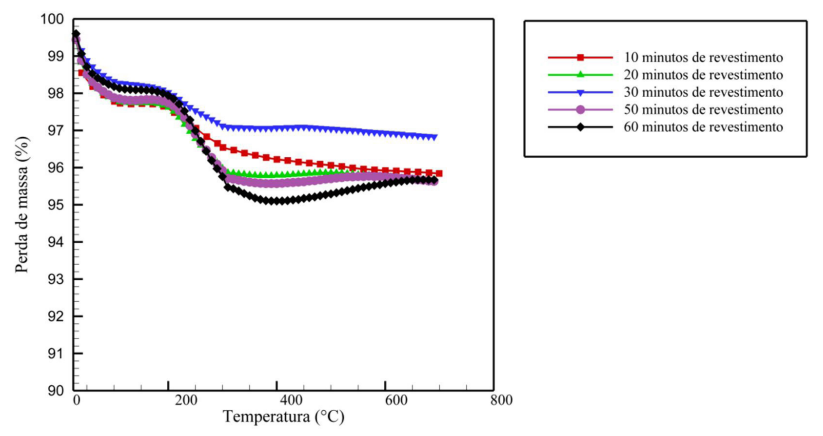

Figura 4. TGA das nanopartículas magnéticas revestidas com PEG.

Como observado na Figura 2, com o incremento no tempo de duração do processo de revestimento das nanopartículas houve um ligeiro aumento no diâmetro médio. As partículas apresentaram um crescimento até o tempo de 30 minutos, após o qual houve um período de estabilização, seguido pelo decréscimo do seu tamanho indicando a ocorrência de uma saturação na camada polimérica que reveste as partículas.

Os valores de DI0, D50 e D90, que descrevem a distribuição granulométrica das partículas, apresentam curvas semelhantes. A parte fina da distribuição, representada por DI0, apresenta tamanhos dentro da faixa ideal para a aplicação desejada. No caso dos valores (D50), embora os valores encontrados para todas as amostras sejam superiores ao esperado, ainda se encontram dentro da faixa desejada para aplicações biomédicas. Com exceção da amostra revestida por 20 minutos, as demais amostras apresentaram um $\mathrm{D} 90$, que representa a medida da parte mais grosseira da distribuição, acima dos valores desejados. Isso pode ser devido à ocorrência de uma pequena aglomeração das nanopartículas apesar do seu revestimento.

A concentração das partículas dispersas em água ultrapura, apresentada na Figura 3, apresentaram curvas semelhantes para os tempos de revestimento indo de 10 a 50 minutos. As partículas que foram revestidas durante 60 minutos apresentaram uma menor concentração, talvez devido à formação de regiões nas quais as nanopartículas formaram pequenos aglomerados. As amostras apresentaram uma ampla distribuição granulométrica devido ao processo manual de maceração das nanopartículas e talvez ao elevado $\mathrm{pH}$ durante a síntese das amostras $(\mathrm{pH}=10)$. $O$ tamanho das amostras pode ser influenciado controlando a temperatura, o $\mathrm{pH}$ e a concentração dos precursores [II].

Os ensaios realizados no TGA/DSC foram feitos mediante atmosfera de nitrogênio para diminuir o efeito de possíveis oxidações nas amostras permitindo que o polímero se decomponha termicamente [19]. Os resultados mostraram a ocorrência de dois estágios para a perda de massa das amostras analisadas. Conforme observado na figura 4 , o primeiro estágio ocorrido antes de $200^{\circ} \mathrm{C}$ foi devido à evaporação de água adsorvida na superfície das partículas. A perda de massa ocorrida no segundo estágio, por volta de $300^{\circ} \mathrm{C}$, refere-se a perda de matéria orgânica PEG (polietilenoglicol) da camada superficial de revestimento que envolve o núcleo magnético das nanopartículas [7], corroborando a existência de uma camada de mistura polimérica adsorvida na superfície do núcleo magnético responsável por garantir a estabilidade ao material. A oxidação do núcleo magnético composto por $\mathrm{Fe}_{3} \mathrm{O}_{4}$ devido à evaporação do PEG produz uma transformação de magnetita $\left(\mathrm{Fe}_{3} \mathrm{O}_{4}\right)$ em maghemita $\left(\gamma-\mathrm{Fe}_{2} \mathrm{O}_{3}\right)[7]$. Uma vez que a queima polimérica 


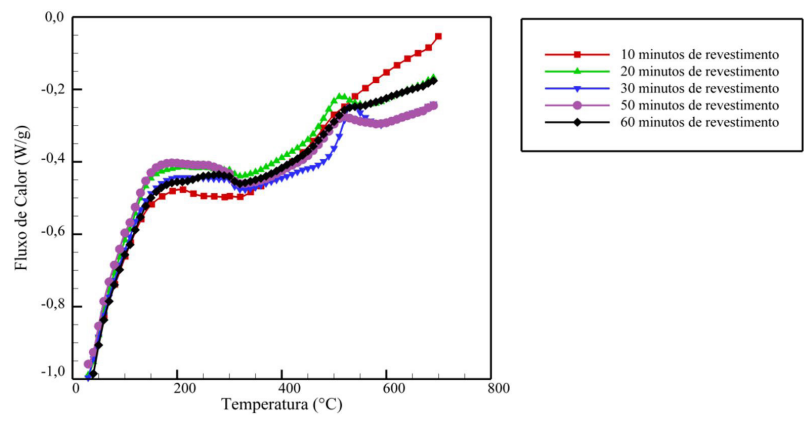

Figura 5. DSC das nanopartículas magnéticas revestidas com PEG.

ocorre a temperaturas elevadas, fica clara a eficiência do uso do PEG na proteção das nanopartículas magnéticas para o propósito biomédico.

O fluxo de calor apresentado na Figura 5 é utilizado para medir as variações de energia térmica empregadas para manter o equilíbrio da temperatura durante um evento térmico. Dessa forma, é possível observar alterações físicas ou químicas da amostra analisada. $O$ pico endotérmico, ocorrido abaixo de $200^{\circ} \mathrm{C}$ é atribuído à desidratação das amostras, ou seja, a perda de água adsorvida na superfície das nanopartículas. $O$ pico exotérmico que ocorre entre $300^{\circ} \mathrm{C}$ e $400^{\circ} \mathrm{C}$ é referente à ocorrência da oxidação da matéria orgânica utilizada na proteção do núcleo de magnetita.

\section{CONCLUSÕES}

Foram realizados variados tempos de recobrimento de nanopartículas de magnetita sintetizadas através da coprecipitação de sais de ferro e revestidas com PEG visando avaliar sua influência no tamanho final das nanopartículas, bem como a eficiência apresentada pelo revestimento empregado na proteção do núcleo magnético, essenciais para o tratamento de hipertermia magnética.

Os resultados obtidos através da análise termogravimétrica confirmaram a adequação do polietilenoglicol (PEG) em revestir as nanopartículas promovendo a proteção do seu núcleo magnético em temperaturas elevadas. A análise da granulometria das amostras mostrou tamanhos médios de nanopartículas aptos para utilização nestas aplicações, bem como o incremento no tamanho apresentado pelas partículas de acordo com o aumento de duração na etapa de revestimento das mesmas indicando a ocorrência de uma saturação da camada de revestimento utilizada para recobrir e funcionalizar as nanopartículas de magnetita. Tempos ótimos de revestimento da ordem de 30 min foram obtidos.

\section{Agradecimentos}

Os autores agradecem a CAPES, CNPq e Faperj.

\section{REFERÊNCIAS}

I Oliveira EM, Paresque MCC, Silva LM, Lopes LCR, Castro JA. Study of the effects of $\mathrm{SiO}_{2}$ nanoparticles concentration on the $\mathrm{TiO}_{2}$ nanoparticles suspensions stabilization. Materials Science Forum. 2016;899:232-236.

2 Shah RR, Davis TP, Glover AL, Nikles DE, Brazel CS. Impact of magnetic field parameters and iron oxide nanoparticle properties on heat generation for use in magnetic hyperthermia. Journal of Magnetism and Magnetic Materials. 2015;387:96-106.

3 Lyutyy TV, Hryshko OM, Yakovenko MY. Uniform and nonuniform precession of a nanoparticle with finite anisotropy in a liquid: opportunities and limitations for magnetic fluid hyperthermia. Journal of Magnetism and Magnetic Materials. 2019;473:198-204.

4 Rani S, Varma GD. Superparamagnetism and metamagnetic transition in Fe3O4 nano-particles synthesized via co-precipitation method at different pH. Physica B, Condensed Matter. 2015;472:66-77.

5 Anbarasu M, Anandan M, Chinnasamy E, Gopinath V, Balamurugan K. Synthesis and characterization of polyethylene glycol (PEG) coated $\mathrm{Fe}_{3} \mathrm{O}_{4}$ nanoparticles by chemical co-precipitation method for biomedical applications. Spectrochimica Acta. Part A: Molecular and Biomolecular Spectroscopy. 201 5; 135:536-539.

6 Zavisova V, Koneracka M, Gabelova A, Svitkova B, Ursinyova M, Kubovcikova M, et al. Effect of magnetic nanoparticles coating on cell proliferation and uptake. Journal of Magnetism and Magnetic Materials. 2019;472:66-73.

7 García-Jimeno S, Estelrich J. Ferrofluid based on polyethylene glycol-coated iron oxide nanoparticles: Characterization and properties. Colloids and Surfaces. A, Physicochemical and Engineering Aspects. 2013;420:74-8I.

8 Ostroverkhov P, Semkina A, Nikitin A, Smirnov V, Vedenyapina D, Vlasova K, et al. Human serum albumin as an effective coating for hydrophobic photosensitizes immobilization on magnetic nanoparticles. Journal of Magnetism and Magnetic Materials. 2019;475: I08-II4.

9 Patsula V, Moskvin M, Dutz S, Horák D. Size-dependent magnetic properties of iron oxide nanoparticles. Journal of Physics and Chemistry of Solids. 2016;88:24-30.

10 Das P, Colombo M, Prosperi D. Recent advances in magnetic fluid hyperthermia for cancer therapy. Colloids and Surfaces. B, Biointerfaces. 2019; I74:42-55. 
I Wang X, Qin M, Fang F, Jia B, Wu H, Qu X, et al. Effect of glycine on one-step solution combustion synthesis of magnetite nanoparticles. Journal of Alloys and Compounds. 2017;719:288-295.

12 Lemal P, Balog S, Geers C, Taladriz-Blanco P, Palumbo P, Hirt AM, et al. Heating behavior of magnetic iron oxide nanoparticles at clinically relevant concentration. Journal of Magnetism and Magnetic Materials. 2019;474:637-642.

13 Gupta AK, Gupta M. Synthesis and surfasse engineering of iron oxide nanoparticles for biomedical applications. Biomaterials. 2005;26:3995-402I.

I4 Dadfar SM, Roemhild K, Drude NI, von Stillfried S, Knüchel R, Kiessling F, et al. Iron oxide nanoparticles: Diagnostic, therapeutic and theranostic applications. Advanced Drug Delivery Reviews. 2019; 138:302-325.

15 Michele FD, Pizzichelli G, Mazzolai B, Sinibaldi E. On the preliminar design of hyperthermia treatments based on infusion and heating of magnetic nanofluids. Mathematical Biosciences. 2016;262:105-1 16.

16 Sanz B, Calatayud MP, Torres TR, Fanarraga ML, Ibarra MR, Goya GF. Magnetic hyperthermia enhances cell toxicity with respect to exogenous heating. Biomaterials. 2017;1 I4:62-70.

17 Harabech M, Leliaert J, Coene A, Crevecoeur G, Roost DV, Dupre L. The effect of the magnetic nanoparticle's size dependence of the relaxation time constant on the specific loss power of magnetic nanoparticle hyperthermia. Journal of Magnetism and Magnetic Materials. 2017;426:206-210.

18 Medeiros SF, Santos AM, Fessi H, Elaissari A. Stimuli-responsive magnetic particles for biomedical applications. International Journal of Pharmaceutics. 201 1;403:139-161.

$19 \mathrm{Si} \mathrm{S,} \mathrm{Kotal} \mathrm{A,} \mathrm{Mandal} \mathrm{TK,} \mathrm{Giri} \mathrm{S,} \mathrm{Nakamura} \mathrm{H,} \mathrm{Kohara} \mathrm{T.} \mathrm{Size-controlled} \mathrm{synthesis} \mathrm{of} \mathrm{magnetite} \mathrm{nanoparticles} \mathrm{in} \mathrm{the}$ presence of polyelectrolytes. Chemistry of Materials. 2004;16:3489-3496.

Recebido em: 23 Jan. 2018

Aceito em: 2 Set. 2019 